\title{
Aggravation de l'anémie et polymorphisme de l'haptoglobine au cours de la drépanocytose au Sénégal
}

\author{
P.M. GUEYE ${ }^{1 *}$, F.GUEYE-TALL ${ }^{1}$, M.SECK ${ }^{2}$, M.O. KANE ${ }^{3}$, R. NDIAYE DIALLO ${ }^{1}$ \\ M. DIATTA ${ }^{1}$, M. SECK ${ }^{4}$, E.M NDOUR ${ }^{1}$, D. DOUPA ${ }^{5}$, B. FAYE ${ }^{2}$, S.DIOP $^{2}$, \\ P. LOPEZ SALL ${ }^{1}$, A. CISSE ${ }^{1}$ et P.A. DIOP ${ }^{1}$ \\ ${ }^{1}$ Laboratoire de Biochimie Pharmaceutique-FMPO, UCAD, Dakar, Sénégal. \\ ${ }^{2}$ Centre National de Transfusion Sanguine, Dakar, Sénégal. \\ ${ }^{3}$ Laboratoire de Physiologie Pharmaceutique-FMPO, UCAD, Dakar, Sénégal. \\ ${ }^{4}$ Laboratoire de Chimie Organique et Thérapeutique-FMPO, UCAD, Dakar, Sénégal. \\ ${ }^{5}$ Laboratoire de Biochimie-UFR des Sciences de la Santé, UGB, Saint-Louis, Sénégal. \\ *Auteur correspondant; E-mail : papa.madieye.gueye@ucad.edu.sn
}

\section{RESUME}

La drépanocytose homozygote s'accompagne d'une augmentation de l'hémoglobine (Hb) plasmatique, susceptible d'exposer les hématies à un stress oxydant. L'haptoglobine présente trois phénotypes majeurs (Hp 1-1, Hp 2-1 et Hp 2-2) susceptibles de fixer l'hémoglobine extracellulaire avec une efficacité différente. L'objectif de ce travail est de voir si la connaissance du phénotype d'Hp pouvait constituer un élément prédictif de l'anémie sévère. Pour cela, il a été recruté 68 drépanocytaires homozygotes, âgés de 5 à 31 ans. Pour chaque patient, un témoin de même sexe et de même âge \pm 2 ans a été recruté. Le phénotypage de l'Hp a été réalisé par électrophorèse sur gel de polyacrylamide. Les résultats du dosage de l'Hb font ressortir que les taux d'Hb sont significativement différentes chez les patients comparées à celles des témoins $(p=0,001)$. Lorsque la répartition a été faite en fonction du phénotype d'Hp, une différence statistiquement significative a été retrouvée entre le phénotype Hp1-1 et le phénotype Hp2-2 ( $<<0,001)$ chez les patients et non chez les témoins. Les résultats de cette étude préliminaire suggéreraient que la connaissance du phénotype d'Hp serait un facteur prédictif de l'anémie sévère au cours de la drépanocytose.

(c) 2014 International Formulae Group. All rights reserved.

Mots clés : Drépanocytose, anémie, phénotypes d'haptoglobine.

\section{INTRODUCTION}

La drépanocytose est une maladie génétique dans laquelle l'hémoglobine A normale $\left(\alpha_{2} \beta_{2}\right)$ est remplacée par l'hémoglobine $\mathrm{S}$, produit d'une mutation sur la chaine $\beta$ de la globine substituant au niveau du $6^{\text {ème }}$ codon une adénine par une thymine, d'où le remplacement d'un acide glutamique par une valine ( $\beta 6 \mathrm{Glu}-\mathrm{Val}$ ) (Bernaudin, 2000).
Cette maladie génétique pose un problème de santé publique dans certains pays, notamment en Afrique. C'est l'hémoglobinopathie la plus répandue au monde. On estime à plus de 50 millions les sujets porteurs du trait drépanocytaire (Galacteros, 2000).

La forme homozygote SS est une maladie hémolytique chronique susceptible de 3 types d'accidents aigus surajoutés: anémies 
graves, infections bactériennes graves et accidents ischémiques vaso-occlusifs (AVO) secondaires aux conflits entre les petits vaisseaux et les drépanocytes (Galacteros, 2000). Des complications organiques et infectieuses diverses peuvent également survenir (Raynal et al., 2007 ; Diop et al., 2010 ; Diagne et al., 2003). Les mécanismes physiopathologiques qui sont à la base de la plupart des complications aussi bien aiguës que chroniques comportent une part importante liée au stress oxydant. Dans ce contexte, la drépanocytose associée à une anémie chronique peut s'accompagner d'une augmentation de l'hémoglobine plasmatique, susceptible d'exposer les globules rouges à un stress oxydant à l'origine d'une aggravation de l'anémie (Buehler et Alayash, 2004).

Des auteurs ont récemment rapporté que l'hémoglobine libre (extra-érythrocytaire) était susceptible d'entraîner des effets délétères sur le globule rouge en termes de stress oxydant pouvant provoquer une hyperhémolyse (Buehler et Alayash, 2004; Gueye et al., 2006 ; Gueye et al., 2010). L'haptoglobine constitue le premier mécanisme de défense de l'organisme contre les effets délétères de l'hémoglobine. En effet, cette protéine fixe de manière stable l'hémoglobine en formant un complexe $\mathrm{Hp}-\mathrm{Hb}$, ce qui inhibe en grande partie sa capacité oxydante. La formation de ce complexe favorise son élimination grâce à la fixation de ce dernier sur le récepteur CD163 des monocytes-macrophages (Kristiansen et al., 2001). L'haptoglobine est un anti-oxydant du fait de sa capacité à prévenir les dommages oxydants dus à l'hémoglobine. Elle présente trois phénotypes majeurs (Hp1-1, Hp 2-1 et Hp2-2), entre lesquels il existe des différences, tant au plan structural qu'au plan fonctionnel (Langlois et Delanghe, 1996). Les différences fonctionnelles sont en rapport avec d'importantes conséquences sur l'évolution de certaines pathologies.

Par ailleurs, il a été rapporté de profondes disparités de sévérité même chez des patients ayant le même phénotype (homozygote SS, hétérozygote composite SC, entre autres), ce qui laisse suggérer que d'autres facteurs génétiques et/ou environnementaux interviennent dans la modulation de la sévérité des complications observées au cours de la drépanocytose (Dossou-Yovo, 2010).

C'est dans ce contexte que nous nous sommes fixés comme objectif de voir si la connaissance du phénotype d'haptoglobine pouvait constituer un élément prédictif de la fréquence et/ou de la sévérité de certaines complications, notamment l'anémie sévère.

\section{MATERIEL ET METHODES \\ Sujets}

Ce travail a porté sur soixante huit (68) patients homozygotes de type SS âgés de 5 à 31 ans, suivis depuis au moins deux ans au niveau du centre National de Transfusion Sanguine (CNTS) avec une bonne observance thérapeutique. Les patients présentant une autre pathologie ont été exclus de l'étude. Au recrutement, sous la supervision du médecin traitant, les patients ont été informés des objectifs de l'étude et ont été recrutés après leur consentement éclairé. Pour chaque patient, un témoin de même sexe et de même âge \pm 2 ans a été recruté parmi les donneurs de sang.

\section{Méthodes}

Les prélèvements sanguins sur EDTA (Ethylene diamine tetraacetic acid), réalisés chez les sujets au repos, à jeun, au niveau du pli du coude ont été immédiatement centrifugés. Les plasmas ont été séparés pour être immédiatement traités ou conservés à $20{ }^{\circ} \mathrm{C}$. Aussi bien chez les patients que chez les témoins, nous avons réalisé les analyses suivantes : phénotypage de l'Hp, numérationformule sanguine (NFS), et dosage de la protéine $\mathrm{C}$ réactive (CRP). La numérationformule sanguine (NFS) a été réalisée à l'aide d'un compteur de globules (Sysmex1000i, Baden, Suisse) et le dosage de la CRP par immunturbidimétrie sur automate A15 (Biosystèmes, Barcelone, Espagne). Le phénotypage de l'haptoglobine a été réalisé selon la méthode de Raymond (Raymond, 1962) par électrophorèse en gel de polyacrylamide à $5 \%$ en utilisant le système 
Mini-Protean-II (Bio-Rad Laboratories, CA, USA) sous $200 \mathrm{~V}$ pendant 2 heures. L'électrophorèse en milieu alcalin a été réalisée en tampon contenant $0,1 \mathrm{~mol} / 1$ de Tris et $0,09 \mathrm{~mol} / \mathrm{l}$ d'acide borique, $\mathrm{pH}$ 8,6. L'électrophorèse à $\mathrm{pH}$ neutre a été réalisée en tampon phosphate $0,03 \mathrm{~mol} / 1, \mathrm{pH} 7,0$.

\section{Analyse statistique}

Les analyses statistiques ont été réalisées grâce au logiciel Statview en utilisant le test de Mann Whitney pour comparer les variables entre patients et témoins et le test de Kruskall Wallis pour déterminer les différences significatives des variables chez les différents types de phénotypes d'haptoglobine. Une valeur de $p$ inférieure à 0,05 a été considérée comme significative.

\section{RESULTATS}

Les phénotypages de l'haptoglobine réalisés avec 68 plasmas de sujets sains révèlent respectivement des fréquences de l'ordre de $27,94 \%, 41,17 \%$, et $30,88 \%$ pour les phénotypes Hp1-1, Hp2-1 et Hp2-2. Chez les sujets de type $\mathrm{SS}$, les fréquences retrouvées sont respectivement de l'ordre de $22,06 \%, 38,23 \%$ et $36,76 \%$ pour les phénotypes Hp1-1; Hp2-1 et Hp2-2. Les résultats de l'étude du polymorphisme de $\mathrm{Hp}$ chez les patients et les témoins sont représentés sur la Figure 1. La comparaison des fréquences dans les deux groupes n'a pas montré de différence statistiquement significative. Trois plasmas de patients n'ont pas pu être classés par électrophorèse en milieu alcalin. Après électrophorèse à $\mathrm{pH}$ neutre, deux se sont révélés être du type 1-1; un seul n'a pas pu être classé et a été assimilé comme ne contenant pas d'haptoglobine décelable.

Les dosages de la CRP ont été effectués pour apprécier l'état stationnaire ou en crise des patients. Les résultats obtenus aussi bien chez les patients que chez les témoins sont répertoriés sur la Figure 2. La comparaison entre les 2 groupes n'a pas montré de différences statistiquement significatives.

Les dosages de l'hémoglobine ont été effectués aussi bien chez les patients que chez les témoins pour apprécier le degré d'anémie. Les résultats représentés sur la Figure 3 font ressortir que les moyennes des taux d'Hb sont significativement différentes chez les patients comparées aux témoins $(\mathrm{p}=0,001)$.

Lorsque la répartition a été faite en fonction $\mathrm{du}$ phénotype $\mathrm{Hp}$, les résultats révèlent chez les témoins (Figure 4) qu'il n y'a pas de différence statistiquement significative des moyennes en $\mathrm{Hb}$ en fonction des trois phénotypes majeurs $(\mathrm{p}=0,88)$. Par contre, chez les patients (Figure 5), une différence statistiquement significative a été retrouvée entre le phénotype Hp1-1 et le phénotype Hp2-2 ( $\mathrm{p}<0,001)$. Les autres comparaisons inter-phénotypes n'ont pas montré de différence statistiquement significative.

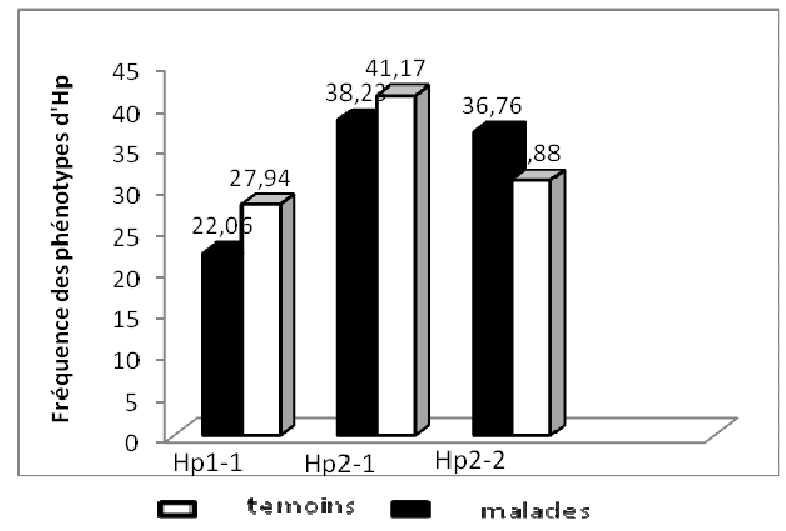

Figure 1 : Fréquences des phénotypes d'haptoglobine chez les malades et les témoins. 


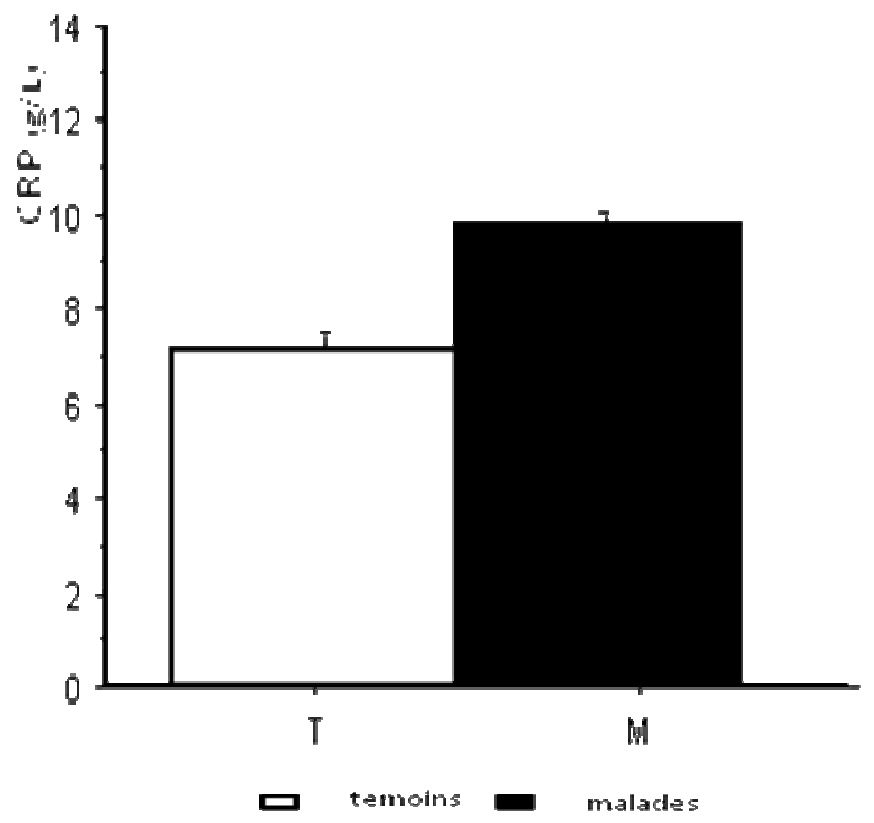

Figure 2 : Concentrations de Protéine C Réactive (CRP) chez les malades et les témoins.

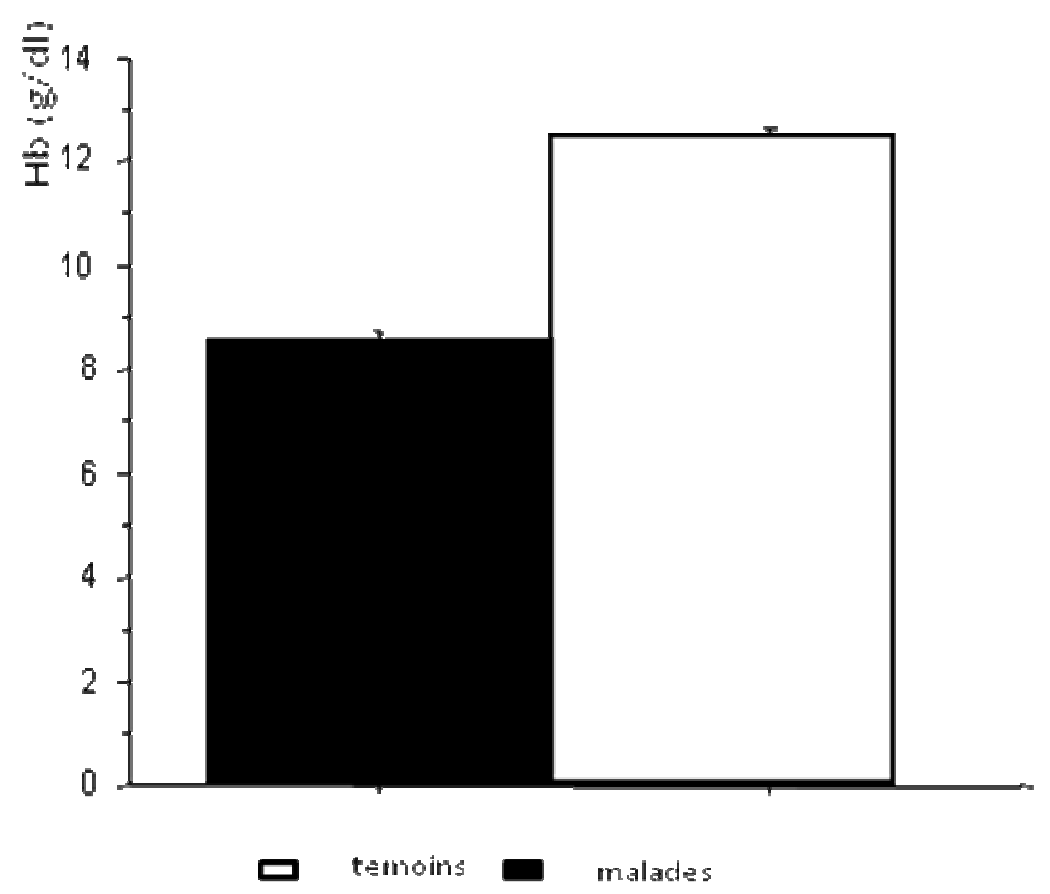

Figure 3 : Taux moyens de l'hémoglobine chez les patients et les témoins. 


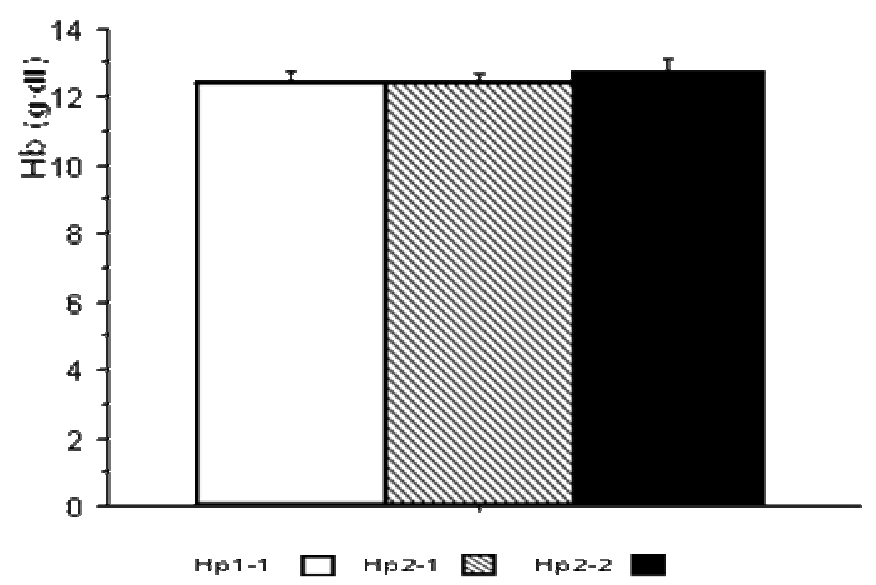

Figure 4 : Taux d'Hb en fonction des phénotypes d'Hp chez les témoins.

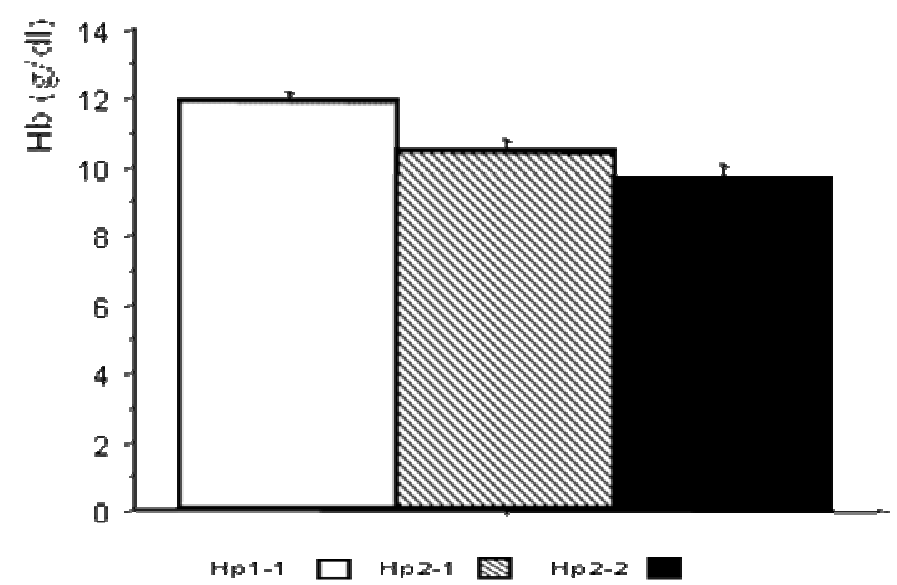

Figure 5 : Sévérité de l'anémie en fonction des phénotypes d'Hp chez les malades.

\section{DISCUSSION}

Le phénotypage de l'haptoglobine réalisé sur 68 plasmas de témoins a montré respectivement des fréquences de l'ordre $28 \%, 41 \%$, et $31 \%$ pour les phénotypes Hp11, Hp2-1 et Hp2-2. Ces fréquences sont relativement différentes de celles retrouvées par Moullec dans une population dakaroise en 1960. Ce dernier avait trouvé $37,19 \%$ pour le Hp1-1, 46,48 \% pour le Hp2-1 et 11,81\% pour le Hp2-2 (Moullec et al., 1960). En effet, on retrouve des fréquences assez variables un peu partout en Afrique, notamment en Gambie, avec $30,57 \%$ de phénotypes Hp1-1, 21,66\% de phénotypes Hp2-1 et 7,01\% de phénotypes Hp2-2 (Harris et al., 1959).
Chez les drépanocytaires de type SS, les fréquences des phénotypes $\mathrm{Hp}$ sont de $22,06 \% ; 38,23 \%$ et $36,76 \%$ respectivement pour les phénotypes Hp1-1, Hp2-1 et Hp2-2. Lorsque ces fréquences sont comparées avec celles retrouvées chez les témoins, nous remarquons une baisse de la fréquence du phénotype Hp1-1 alors que celle du Hp2-2 augmente. Toutefois, les différences observées n'ont pas été significatives.

Lors du recrutement, nous avons utilisé le dosage de la CRP pour trier les patients en phase stationnaire de ceux en état de crise drépanocytaire. Les résultats obtenus montrent une légère augmentation chez les patients comparés aux témoins. Toutefois, les 
différences observées n'ont pas été statistiquement significatives.

Dans notre étude, un plasma d'un patient type SS n'a présenté aucune bande caractéristique d'Hp (possédant une activité peroxydasique) après électrophorèse en présence d'hémoglobine ni à $\mathrm{pH}$ alcalin, ni à $\mathrm{pH}$ neutre nous amenant à classer le patient dans le groupe de sujets à Hp non détectable. Un tel fait pourrait faire penser qu'il s'agit d'un individu atteint d'hypo voire d'anhaptoglobinémie. En effet, des cas, bien que rares (1/1000) de déficit congénital en haptoglobine, ont été rapportés (Koda et al., 2000). Par contre, des fréquences d'anhaptoglobinémie plus élevées ont été rapportées en Australie (1,7\%), ainsi que chez des populations noires $(30 \%)$ en Afrique du sud, au Cameroun et au Nigeria (Delanghe et al., 1998). A ce titre, il est important de différencier l'anahptoglobinémie (phénotype Hp 0-0) qui est due à l'absence d'expression du gène de l'Hp (Langlois et Delanghe, 1996 ; Koda, 2000), des hypohaptoglobinémies qui peuvent être secondaires à une maladie congénitale comme les désordres hémolytiques (drépanocytose, déficit en glucose-6-phosphate-déshydrogénase, thalassémie. (Atkinson SH, 2006).

Les résultats de la détermination du taux d'Hb chez les patients de type SS comparés aux témoins ont montré une diminution significative. Ces résultats sont en accord avec des données rapportées par plusieurs auteurs, qui relatent l'existence d'une anémie chronique chez les sujets drépanocytaires de type SS (Bernaudin, 2000, Galacteros, 2000).

Chez les patients de type SS, les sujets de phénotype Hp2-2 présentaient des taux moyens d'Hb significativement plus bas comparés à ceux retrouvés chez les patients Hp1-1 et Hp2-1. Les résultats obtenus pourraient s'expliquer en partie par le fait que l'hémoglobine $\mathrm{S}$ constitue un facteur de risque de peroxydation pour la membrane érythrocytaire (Silva et al., 2013). Ainsi, chez ces patients, la peroxydation lipidique au niveau des membranes des globules rouges jouerait un rôle dans l'aggravation de l'anémie. Dans une étude antérieure (Gueye et al., 2006), nous avons montré que l'hémoglobine extra-érythrocytaire pouvait induire, sur le globule, un stress oxydant se traduisant par une lyse précoce. En effet, en dehors du globule rouge et de son contenu antioxydant, la toxicité oxydative de l'Hb s'affirme et va produire, via la réaction de Fenton, des radicaux libres qui vont attaquer la membrane des globules rouges et entraîner leur lyse précoce (Silva et al., 2013). L'action anti-oxydante de l'haptoglobine étant phénotype-dépendante (Asleh et al., 2005 ; Gueye et al., 2006), cela pourrait expliquer en partie le fait que l'anémie retrouvée chez les patients de type SS soit plus intense chez les patients de type Hp2-2 comparée à celle retrouvée au niveau des 2 autres phénotypes. En effet, ce phénotype étant moins efficace dans la fixation de l'Hb et la neutralisation de son pouvoir oxydant, les patients concernés seraient plus prédisposés à une aggravation de l'anémie comparée aux sujets des autres phénotypes. Cependant, ces résultats doivent être interprétés avec prudence, d'autant plus que d'autres facteurs comme le taux d'Hb fœtale pourraient intervenir dans la modulation de la sévérité des signes cliniques (Nacoulma et al., 2006).

\section{Conclusion}

$\mathrm{Au}$ cours de ce travail préliminaire, nous avons étudié l'influence du polymorphisme de l'haptoglobine dans la survenue d'une anémie grave chez des patients drépanocytaires. Les résultats obtenus sont en faveur d'une prédisposition des sujets de phénotype Hp2-2 à présenter une aggravation de l'anémie. Cette étude préliminaire devrait être complétée par la réalisation d'une étude à plus grande échelle, afin de confirmer ces résultats obtenus à partir 
d'un faible échantillonnage. Une évaluation des marqueurs de peroxydation lipidique comme les substances réagissant avec l'acide thiobarbiturique (TBARS) et la détermination de l'activité des enzymes du globule rouge ainsi que la prise en compte d'autres facteurs comme le taux d'Hb fœtale devraient permettre d'élucider les mécanismes de protection de l'haptoglobine vis-à-vis de la survenue d'une aggravation de l'anémie. L'ensemble de ces résultats pourrait éventuellement permettre d'envisager une stratégie thérapeutique notamment par l'utilisation d'antioxydants chez les patients à risque.

\section{REFERENCES}

Bernaudin F. 1999 Résultats et indications actuelles de l'allogreffe de la moelle dans la drépanocytose. Path Biol., 47: 59-64.

Galacteros F. 2000. Drépanocytose. Encyclopedie Orphaned, 3: 351-360.

Raynal G, Bracq A, Tillou X, Limani K, Petit J. 2007. Les complications rénales de la drépanocytose. Progrès en Urologie, 17 : 794-795.

Diop S, Diop D, Seck M, Guèye Y, Faye A, Dièye TN, Touré Fall AO, Sall Diallo A, Thiam D, Diakhaté L. 2010. Predictive factors of chronic complications in adult sickle cell anemia patients in Dakar, Senegal. Med Trop., 70(5-6): 471-474.

Diagne I, Gueye NDR, Signaté-Sy H, Camara B, Lopez-Sall P, MBaye D, Sarr AM, Ba M, Sow HD, Kuakuvi N. 2003. Prise en charge de la drépanocytose chez l'enfant en Afrique : expérience de la cohorte de l'HEAR de Dakar. Med Trop., 63: 513520.

Buehler PW, Alayash AI. 2004. Toxicities of hemoglobin solutions: in search of invitro and in-vivo model systems. Transfusion, 44(10): 1516-1530.

Gueye PM, Glasser N, Ferard G, Lessinger JM. 2006. Influence of human haptoglobin polymorphism on oxidative stress induced by free hemoglobin on red blood cells. Clin Chem Lab Med., 44(5): 542-547.

Gueye PM, Bertrand F, Duportail G, Lessinger JM. 2010. Extracellular haemoglobin, oxidative stress and quality of red blood cells relative to perioperative blood salvage. Clin. Chem. Lab. Med., 48(5): 677-83.

Kristiansen M, Graversen JH, Jacobsen C, Sonne O, Hoffman HJ, Law SK, Moestrup SK. 2001. Identification of the hemoglobin scavenger receptor. Nature, 409: 198-201.

Langlois MR, Delanghe JR. 1996. Biological and clinical significance of haptoglobin polymorphism in humans. Clin. Chem., 42(10): 1589-1600.

Dossou-Yovo OP. 2010. Gènes Modificateurs De l'Expression Phénotypique de la Drépanocytose : Modulation Clinique de la Drépanocytose. Editions Universitaires Européennes: United Kingdom.

Raymond S. 1962. A convenient apparatus for vertical gel electrophoresis. Clin. Chem., 8: $455-470$.

Moullec J, Fine JM, Linhard J. 1960 Les groupes d'haptoglobine dans un échantillon de population africaine de Dakar. Revue d'Hématologie, 15: 174182.

Harris H, Robson EB, Siniscalco M. 1959. Genetics of the Plasma Protein Variants. Biochemistry of Human Genetics, Ciba Foundation Symposium. Churchill, Edition: Londres; 151.

Koda Y, Watanabe Y, Soejima M, Shimada E, Nishimura M, Morishita K, Moriya S , Mitsunaga S, Tadokoro K, Kimura $\mathrm{H}$. 2000. Simple PCR detection of haptoglobin gene deletion in anhaptoglobinemic patients with antihaptoglobin antibody that causes anaphylactic transfusion reactions. Blood, 95(4): 1138-1143. 
Delanghe J, Langlois M, De Buyzere. 1998. Congenital anhaptoglobinemia versus acquired hypohaptoglobinemia. Blood, 91(9): 3524.

Atkinson SH, Rockett K, Sirugo G, Bejon PA, Fulford A, O'Connell MA, Bailey R, Kwiatkowski DP, Prentice AM. 2006. Seasonal childhood anaemia in West Africa is associated with the haptoglobin 2-2 genotype. Plos Med., 3(5): 652-658.

Silva DG, Belini JE, De Almeida EA, BoniniDomingos CR. 2013. Oxidative stress in sickle cell disease: an overview of erythrocyte redox metabolism and current antioxidant therapeutic strategies Free Radic. Biol. Med., 65: 1101-1109.

Asleh R, Guetta J, Litman SK, Lotan RM, Levy AP. 2005. Haptoglobin genotypeand diabetes-dependent differences in iron-mediated oxidative stress in vitro and in vivo. Circ Res., 96(4) : 435-441.

Nacoulma EVC, Sawadogo D, Sakandé J, Sangaré A, Sess ED. 2006. Influence de l'hémoglobine fotale sur le stress oxydant chez le drépanocytaire homozygote vivant à Abidjan, Côte d'Ivoire. Bull. Soc. Path. Exo., 99: 241244. 